August, 1997

\title{
A Possible Unification Model for All Basic Forces
}

\author{
K. C. $\mathrm{Chou}^{a}$ and Y. L. Wu ${ }^{b}$ \\ ${ }^{a}$ Chinese Academy of Sciences \\ Beijing 100086 P.R. China \\ ${ }^{b}$ Institute of Theoretical Physics \\ Chinese Academy of Sciences, P.O. Box 2735 \\ Beijing, 100080, P.R. China
}

*Supported in part by Outstanding Young Scientist Fund of China 
AS-ITP-97-22

\title{
A Possible Unification Model for All Basic Forces
}

\author{
K. C. $\mathrm{Chou}^{a}$ and Y. L. Wu ${ }^{b \dagger}$ \\ ${ }^{a}$ Chinese Academy of Sciences \\ Beijing 100086 P.R. China \\ ${ }^{b}$ Institute of Theoretical Physics \\ Chinese Academy of Sciences, P.O. Box 2735 \\ Beijing, 100080, P.R. China
}

(August 1997)

\begin{abstract}
A unification model for strong, electromagnetic, weak and gravitational forces is proposed. The tangent space of ordinary coordinate 4-dimensional spacetime is a submanifold of an 14-dimensional internal spacetime spanned by four frame fields. The unification of the standard model with gravity is governed by gauge symmetry in the internal spacetime.
\end{abstract}

PACS numbers: 12.10.Gq, 04.50.+h, 11.30.Ly

Typeset using REVTEX

†Supported in part by Outstanding Young Scientist Fund of China 
One of the great theoretical endeavours in this century is to unify gravitational force characterized by the general relativity of Einstein [1] with all other elementary particle forces (strong, electromagnetic and weak ) described by Yang-Mills gauge theory [2]. One of the difficulties arises from the no-go theorem [3] which was proved based on a local Elativistic quantum field theory in 4-dimensional spacetime. Most of the attempts to unify all basic forces involve higher dimensional spacetime, such as Kaluza-Klein Yang-Mills theories 《⿻ supergravity theories [5] and superstring theories [6], etc. In the Kaluza-Klein Yang-Mills theories, in order to have a standard model gauge group as the isometry group of the manifold, the minimal number of total dimensions has to be eleven [7]. Even so, the Kaluza-Klein approach is not rich enough to support the fermionic representations of the standard model due to the requirement of the Atiyah-Hirzebruch index theorem. The maximum supergravity has $\mathrm{SO}(8)$ symmetry, its action is usually also formulated as an $\mathrm{N}=1$ supergravity theory in eleven-dimensional spacetime. Unfortunately, the $\mathrm{SO}(8)$ symmetry is too small to include the standard model. Consistent superstring theories have also been built based on ten-dimensinal spacetime. In superstring theories, all the known particle interactions can be reproduced, but millions of vacua have been found. The outstanding problem is to find which one is the true vacuum of the theory.

In this note, we will consider an alternative scheme. Firstly, we observe that quarks and leptons in the standard model [8] can be unified into a single 16-dimensional representation of complex chiral spinors in $\mathrm{SO}(10)$ [9]. Each complex chiral spinor belong to a single 4-dimensional representation of $\mathrm{SO}(1,3)$. In an unified theory, it is an attractive idea to treat these 64 real spinor components on the same footing, i.e., they have to be a single representation of a larger group. It is therefore natural to consider $\mathrm{SO}(1,13)$ as our unified group and the gauge potential of $\mathrm{SO}(1,13)$ as the fundamental interaction that unifies the four basic forces (strong, electromagnetic, weak and gravitational) of nature. Secondly, to avoid the restriction given by no-go theorem and other problems mentioned above, we consider the ordinary coordinate spacetime remains to be a 4-dimensional manifold $S_{4}$ with metric $g_{\mu \nu}(x), \mu, \nu=0,1,2,3$. At each point $\mathrm{P}: x^{\mu}$, there is an d-dimensional flat space $M_{d}$ 
with $d>4$ and signature $(1,-1, \cdots,-1)$. We assume that the tangent space $T_{4}$ of $S_{4}$ at point $\mathrm{P}$ to be an 4-dimensional submanifold of $M_{d}$ spanned by four vectors $e_{\mu}^{A}(x) \mu=0,1,2,3$; $A=0,1, \cdots, d-1$ such that

$$
g_{\mu \nu}(x)=e_{\mu}^{A}(x) e_{\nu}^{A}(x) \eta_{A B}
$$

where $\eta_{A B}=\operatorname{diag} .(1,-1, \cdots,-1)$ can be considered as the metric of the flat space $M_{d}$. We shall call $e_{\mu}^{A}(x)$ to be the generalized vierbein fields or simply the frame fields. Once the the frame fields $e_{\mu}^{A}(x)$ are given, we can always supplement with another d-4 vector fields $e_{m}^{A}(x) \equiv e_{m}^{A}\left(e_{\mu}^{A}(x)\right), m=1,2, \cdots, d-4$, such that

$$
e_{\mu}^{A}(x) e_{m}^{B}(x) \eta_{A B}=0, \quad e_{m}^{A}(x) e_{n}^{B}(x) \eta_{A B}=g_{m n}
$$

where $g_{m n}=\operatorname{diag} .(-1, \cdots,-1) . e_{m}^{A}(x)$ can be uniquely determined up to an $\mathrm{SO}(\mathrm{d}-4)$ rotation. In the flat manifold $M_{d}$ we can use $e_{\mu}^{A}(x)$ and $e_{m}^{A}(x)$ to decompose it into two orthogonal manifolds $T_{4} \otimes C_{d-4}$. Where $C_{d-4}$ will consider to be the internal space describing $\mathrm{SO}(\mathrm{d}-4)$ internal symmetry besides the spin and is spanned by the d-4 orthonormal vectors $e_{m}^{A}(x)$. In the new frame system of $M_{d}$ the metric tensor is of the form

$$
\left(\begin{array}{cc}
g_{\mu \nu}(x) & 0 \\
0 & g_{m n}
\end{array}\right)
$$

With $e_{\mu}^{A}(x)$ and $e_{m}^{A}(x)$, we can now define the covariant vectors as $e_{A}^{\mu}(x)$ and $e_{A}^{m}(x)$ satisfying

$$
\begin{array}{ll}
e_{A}^{\mu}(x) e_{\nu}^{A}(x)=g_{\nu}^{\mu}, & e_{A}^{m}(x) e_{n}^{A}(x)=g_{n}^{m} \\
e_{A}^{\mu}(x) e_{m}^{A}(x)=0, & e_{A}^{m}(x) e_{\mu}^{A}(x)=0
\end{array}
$$

Under general coordinate transformations and the rotations in $M_{d}, e_{\mu}^{A}(x)$ transform as a covariant vector in ordinary coordinate spactime and a vector in the $M_{d}$ rotation, $e_{m}^{A}(x)$ transform as a covariant vector in the $C_{d-4}$ rotation and a vector in the $M_{d}$ rotation. For a theory to be invariant under both general coordinate transformations and local rotations 
in the flate space $M_{d}$, it is necessary to introduce affine connection $\Gamma_{\mu \nu}^{\rho}(x)$ for general coordinate transformations and gauge potential $\Omega_{\mu}^{A B}(x)=-\Omega_{\mu}^{B A}(x)$ for d-dimensional rotation $\mathrm{SO}(1, \mathrm{~d}-1)$ in $M_{d}$. These transformations are connected by the requirement that $T_{4}$ has to be the submanifold of $M_{d}$ spanned by four vectors $e_{\mu}^{A}(x)$ at point $\mathrm{P}$ and $e_{\mu}^{A}(x)$ should be a covariantly constant frame and satisfy the condition

$$
D_{\mu} e_{\rho}^{A}=\partial_{\mu} e_{\rho}^{A}-\Gamma_{\mu \rho}^{\sigma} e_{\sigma}^{A}+g_{U} \Omega_{\mu B}^{A} e_{\rho}^{B}=0
$$

It is then easily verified that

$$
\begin{aligned}
& D_{\mu} g_{\rho \sigma}=\partial_{\mu} g_{\rho \sigma}-\Gamma_{\mu \rho}^{\lambda} g_{\lambda \sigma}-\Gamma_{\mu \sigma}^{\lambda} g_{\rho \lambda}=0 \\
& D_{\mu} e_{A}^{\rho}=\partial_{\mu} e_{A}^{\rho}+\Gamma_{\mu \sigma}^{\rho} e_{A}^{\sigma}-g_{U} \Omega_{\mu A}^{B} e_{B}^{\rho}=0
\end{aligned}
$$

With the above considerations, we can now construct an invariant action under general coordinate transformations in the ordinary coordinate spacetime and the local $\mathrm{SO}(1, \mathrm{~d}-1)$ group symmetry in $M_{d}$ with eq. (5) as a constraint. In addition, the action is required to have no dimensional parameters and to be renormalizable in the sense of the power counting. The general form of the action which satisfies these requirements is

$$
\begin{aligned}
S_{B} & =\int d^{4} x \sqrt{-g}\left\{-\frac{1}{4} F_{\mu \nu}^{A B} F_{\rho \sigma}^{C D} g^{\mu \rho} g^{\nu \sigma} \eta_{A C} \eta_{B D}\right. \\
& -\frac{1}{2} \xi \phi^{2} F_{\mu \nu}^{A B} e_{A}^{\mu} e_{B}^{\nu}+\frac{1}{2} g^{\mu \nu} \partial_{\mu} \phi \partial_{\nu} \phi+\frac{1}{4} \lambda \phi^{4} \\
& +\zeta F_{\mu \nu}^{A B} F_{\rho \sigma}^{C D} g^{\mu \rho} \eta_{A C} e_{B}^{\nu} e_{D}^{\sigma}+a_{1} F_{\mu \nu}^{A B} F_{\rho \sigma}^{C D} e_{C}^{\mu} e_{D}^{\nu} e_{A}^{\rho} e_{B}^{\sigma} \\
& \left.+a_{2} F_{\mu \nu}^{A B} F_{\rho \sigma}^{C D} e_{C}^{\mu} e_{B}^{\nu} e_{A}^{\rho} e_{D}^{\sigma}+a_{3} F_{\mu \nu}^{A B} F_{\rho \sigma}^{C D} e_{A}^{\mu} e_{B}^{\nu} e_{C}^{\rho} e_{D}^{\sigma}\right\}
\end{aligned}
$$

where $\phi(x)$ is a scalar field introduced to avoid the dimensional coupling constants. $a_{i}$ $(\mathrm{i}=1,2,3), \zeta, \xi$ and $\lambda$ are dimensionless parameters. $F_{\mu \nu}^{A B}$ is the field strength defined in a standard way

$$
F_{\mu \nu}^{A B}=\partial_{\mu} \Omega_{\nu}^{A B}-\partial_{\nu} \Omega_{\mu}^{A B}+g_{U}\left(\Omega_{\mu C}^{A} \Omega_{\nu}^{C B}-\Omega_{\nu C}^{A} \Omega_{\mu}^{C B}\right)
$$

The tensor $F_{\mu}^{A}$ is defined as $F_{\mu}^{A}=F_{\mu \nu}^{A B} e_{B}^{\nu}$ 
Using the frame fields $e_{A}^{\mu}(x)$ and $e_{A}^{m}\left(e_{\mu}^{A}(x)\right)$, we can decompose $\Omega_{\mu}^{A B}(x)$ into three parts $e_{A}^{\sigma}(x) \Omega_{\mu}^{A B}(x) e_{B}^{\rho}(x)(\rho, \sigma=0,1,2,3)$ which describe the gravity, and $e_{A}^{m}(x) \Omega_{\mu}^{A B}(x) e_{B}^{n}(x)$ which characterize gauge interactions, as well as $e_{A}^{m}(x) \Omega_{\mu}^{A B}(x) e_{B}^{\sigma}(x)$ which connect gravity with gauge interactions. From the constraints of eq.(5), we obtain

$$
\begin{aligned}
& g_{U} e_{\sigma A}(x) \Omega_{\mu}^{A B}(x) e_{B}^{\rho}(x)=\Gamma_{\mu \sigma}^{\rho}-e_{\sigma A} \partial_{\mu} e^{\rho A} \\
& g_{U} e_{m A}(x) \Omega_{\mu}^{A B}(x) e_{B}^{\sigma}(x)=-e_{m A} \partial_{\mu} e^{\sigma A}
\end{aligned}
$$

Similarly, we can reespress $e_{A}^{m}(x) \Omega_{\mu}^{A B}(x) e_{B}^{n}(x)$ as

$$
e_{A}^{n}(x) \Omega_{\mu}^{A B}(x) e_{B}^{m}(x)=A_{\mu}^{m n}(x)-\frac{1}{2}\left(e_{A}^{n} \partial_{\mu} e^{m A}-e_{A}^{m} \partial_{\mu} e^{n A}\right)
$$

where $A_{\mu}^{m n}(x)=-A_{\mu}^{n m}(x)(\mathrm{m}, \mathrm{n}=1, \cdots, \mathrm{d}-4)$ is the gauge potential for (d-4)-dimensional rotation $\mathrm{SO}(\mathrm{d}-4)$ in $C_{d-4}$.

Note that not all the gauge fields $\Omega_{\mu}^{A B}(x)$ are simply new propagating fields due to the constraints $D_{\mu} e_{\rho}^{A}=0$. By counting the constraint equations $(4 \times 4 \times \mathrm{d})$, unknowns $\Omega_{\mu}^{A B}(x)$ (with $4 \mathrm{~d}(\mathrm{~d}-1) / 2$ degrees of freedom) and $e_{\mu}^{A}(x)$ (with $4 \times \mathrm{d}$ degrees of freedom) as well as $\Gamma_{\mu \sigma}^{\rho}$ (with 40 degrees of freedom for the symmetric parts $\Gamma_{(\mu \sigma)}^{\rho}=\Gamma_{(\sigma \mu)}^{\rho}$ and 24 degrees of freedom for antisymmetric parts $\left.\Gamma_{[\mu \sigma]}^{\rho}=-\Gamma_{[\sigma \mu]}^{\rho}\right)$, one sees that besides the antisymmetric parts $\Gamma_{[\mu \sigma]}^{\rho}$, the independent degrees of freedom are $(4 \mathrm{~d}+4(\mathrm{~d}-4)(\mathrm{d}-5) / 2)$. These independent degrees of freedom coincide with the degrees of freedom of the frame fields $e_{\mu}^{A}(x)$ and the gauge fields $A_{\mu}^{m n}(x)$ of the group $\mathrm{SO}(\mathrm{d}-4)$. In addition, the gauge conditions in the coset $\mathrm{SO}(1, \mathrm{~d}-$ 1)/ $\mathrm{SO}(\mathrm{d}-4)$ lead to additional constraints (4d-10). Thus the independent degrees of freedom are reduced to $(10+4(\mathrm{~d}-4)(\mathrm{d}-5) / 2)$ which exactly match with the degrees of freedom of the metric tensor $g_{\mu \nu}(x)$ and the gauge fields $A_{\mu}^{m n}(x)$ of the group $\mathrm{SO}(\mathrm{d}-4)$. For $\mathrm{d}=14$, the resulting independent degrees of freedom of the fields are sufficient to describe the four basic forces. Where the general relativity of the Einstein theory is described by the metric tensor. Photon, W-bosons and gluons, that mediate the electromagnetic, weak and strong interactions respectively, are different manifestations of the gauge potential $A_{\mu}^{m n}(x)$ of the symmetry group $\mathrm{SO}(10)$ [9]. The curvature tensor $R_{\mu \nu \sigma}^{\rho}$ and the Ricci tensor $R_{\nu \sigma}=R_{\mu \nu \sigma}^{\rho} g_{\rho}^{\mu}$ 
as well as the scalar curvature $R=R_{\nu \sigma} g^{\nu \sigma}$ are simply related to the field strength $F_{\mu \nu}^{A B}$ via $R_{\mu \nu \sigma}^{\rho}=g_{U} F_{\mu \nu}^{A B} e_{A}^{\rho} e_{\sigma B}, R_{\nu \sigma}=g_{U} F_{\mu \nu}^{A B} e_{A}^{\mu} e_{\sigma B}$ and $R=g_{U} F_{\mu \nu}^{A B} e_{A}^{\mu} e_{B}^{\nu}$. It is not difficult to check that $R_{\mu \nu}^{A B} R_{A B}^{\mu \nu}=F_{\mu \nu}^{m n} F_{m n}^{\mu \nu}+g_{U}^{-2} R_{\mu \rho \nu \sigma} R^{\mu \rho \nu \sigma}$, and $R_{\mu}^{A} R_{A}^{\mu}=g_{U}^{-2} R_{\mu \rho} R^{\mu \rho}$. Where $F_{\mu \nu}^{m n}(x)$ is the field strength of the gauge potential $A_{\mu}^{m n}(x)$

$$
F_{\mu \nu}^{m n}=\partial_{\mu} A_{\nu}^{m n}-\partial_{\nu} A_{\mu}^{m n}+g_{U}\left(A_{\mu q}^{m} A_{\nu}^{q n}-A_{\nu q}^{m} A_{\mu}^{q n}\right)
$$

With these relations, the action $S_{B}$ can be simply reexpressed as

$$
\begin{aligned}
S_{B} & =\int d^{4} x \sqrt{-g}\left\{-\frac{1}{4} F_{\mu \nu}^{m n} F_{m n}^{\mu \nu}+\frac{1}{2} \partial_{\mu} \phi \partial^{\mu} \phi+\frac{1}{4} \lambda \phi^{4}-\frac{1}{2} \xi g_{U}^{-1} \phi^{2} R\right. \\
& \left.+g_{U}^{-2}\left[\left(a_{1}-\frac{1}{4}\right) R_{\mu \rho \nu \sigma} R^{\mu \rho \nu \sigma}+\left(a_{2}+\zeta\right) R_{\mu \rho} R^{\mu \rho}+a_{3} R^{2}\right]\right\}
\end{aligned}
$$

which has the same form as the action of a multiplicatively renormalized unified gauge theory including so-called $R^{2}$-gravity and a renormalizable scalar matter field as well a nonminimal gravitational-scalar coupling.

In the real world, there exist three generations of quarks and leptons. Each generation of the quarks and leptons have 64 real degrees of freedom. These degrees of freedom will be represented by the 64 compotents of a single Weyl fermion $\Psi_{+}(x)$ belonging to the fundamental spinor representation of $\mathrm{SO}(1,13)$. The action for fermions is given by

$$
S_{F}=\int d^{4} x \sqrt{-g}\left\{\frac{1}{2} \bar{\Psi}_{+} e_{A}^{\mu} \Gamma^{A}\left(i \partial_{\mu}+g_{U} \Omega_{\mu}^{B C} \frac{1}{2} \Sigma_{B C}\right) \Psi_{+}+\text {h.c. }\right\}
$$

where $\Sigma_{A B}$ are the generators of the $\mathrm{SO}(1, \mathrm{~d}-1)$ in the spinor representations and given by $\Sigma_{A B}=\frac{i}{4}\left[\Gamma_{A}, \Gamma_{B}\right] . \Gamma^{A}$ are the gamma matrices that obey $\left\{\Gamma^{A}, \Gamma^{B}\right\}=2 \eta^{A B}$. Note that the resulting total action $S=S_{B}+S_{F}$ is simple, but it is nontrival for fermionic interactions since the gauge potentials $\Omega_{\mu}^{A B}(x)$ are related to the independent degrees of freedom $A_{\mu}^{m n}(x)$ and $e_{\mu}^{A}(x)$ by some nontrival relations given in eqs.(10), (11) and (12). In particular, the supplemented frame fields $e_{m}^{A}(x)$ will have a highly nonlear dependence on the frame fields $e_{\mu}^{A}(x)$

Now let us consider the conservation laws under the general coordinate transformations and local rotation $\mathrm{SO}(1, \mathrm{~d}-1)$. Under the local rotation $\Psi_{+}(x) \rightarrow e^{-\frac{1}{2} i \omega^{A B} \Sigma_{A B}} \Psi_{+}(x)$, it is not difficult to find the conservation law as 


$$
D_{\mu}\left(\sqrt{-g} S_{A B}^{\mu}\right)-\sqrt{-g} T_{[A B]} \equiv 0
$$

with

$$
\begin{aligned}
S_{A B}^{\mu} & =g_{U} \frac{1}{4} \bar{\Psi}_{+} e_{C}^{\mu}\left\{\Gamma^{C}, \Sigma_{A B}\right\} \Psi_{+} \\
T_{[A B]} & =-i \frac{1}{2}\left[\bar{\Psi}_{+} e_{A}^{\mu} \Gamma_{B} D_{\mu} \Psi_{+}-\left(D_{\mu} \bar{\Psi}_{+}\right) e_{A}^{\mu} \Gamma_{B} \Psi_{+}\right]
\end{aligned}
$$

The general coordinate transformations lead to the well-known energy-momentum conservation law as

$$
D^{\nu}\left(\sqrt{-g} T_{\mu \nu}\right) \equiv \sqrt{-g} F_{\mu \nu}^{A B} S_{A B}^{\nu}
$$

with

$$
T_{\mu \nu}=g_{\mu \nu} \mathcal{L}-i \frac{1}{2} e_{\nu}^{A}\left[\bar{\Psi}_{+} \Gamma_{A} D_{\mu} \Psi_{+}-\left(D_{\mu} \bar{\Psi}_{+}\right) \Gamma_{A} \Psi_{+}\right]
$$

Using the covariantly constant frame fileds $e_{\mu}^{A}(x)$, we can project $S_{A B}^{\mu}$ and $T_{[A B]}$ into

$$
\begin{aligned}
S_{\rho \sigma}^{\mu} & =S_{A B}^{\mu} e_{\rho}^{A} e_{\sigma}^{B}, \\
T_{[\rho \sigma]} & =T_{[A B]} e_{\rho}^{A} e_{\sigma}^{B}=T_{\rho \sigma}-T_{\sigma \rho}
\end{aligned}
$$

The angular momentum conservation law becomes

$$
D_{\mu}\left(\sqrt{-g} S_{\rho \sigma}^{\mu}\right)-\sqrt{-g} T_{[\rho \sigma]} \equiv 0
$$

It is then easy to show that these two conservation laws (eqs.(19) and (23)) are essentially the same as occurs in special relativity by noticing the following relations

$$
-T_{[\rho \sigma]}=\partial_{\mu} L_{[\rho \sigma]}^{\mu} \equiv \partial_{\mu}\left(x_{\rho} T_{\sigma}^{\mu}-x_{\sigma} T_{\rho}^{\mu}\right)
$$

Here $L_{\rho \sigma}^{\mu}$ is the orbital angular momentum tensor and $J_{\rho \sigma}^{\mu} \equiv S_{\rho \sigma}^{\mu}+L_{\rho \sigma}^{\mu}$ represents the total angular momentum tensor.

From simple ideas we have provided a unification model for strong, electromagnetic, weak and gravitational forces and constructed the action without dimensional parameters 
as the basis for quantum theory of all the basic forces of the elementary particles. Such a theory is conjectured to be multiplicative renormalizable though it may remain an effective theory of a more fundamental theory. One can find a formal proof of the renormalizability of the $R^{2}$-gravity with a scalar field in [10]. It was known that in the general relativity only the Einstein equations have been tested to be in good with known experimental data at the classical level. Thus the general relativity of the Einstein theory may be interpreted as a classical theory in the low energy limit, so that the Einstein-Hilbert and cosmological terms may be induced as a result of the low energy limit [10,11]. For instance, these terms may result from a spontaneous symmetry breaking. We hope that the present model has provided us a new insight for unifying all the basic forces within the framework of quantum field theory. Though the ideas and the resulting model are both simple, there remains more theoretical work and experimental efforts needed to test whether they are the true choice of nature. 


\section{REFERENCES}

[1] A. Einstein, Sitz. Preuss. Akad. Wiss. 778, 844, (1915).

[2] C.N. Yang and R.L. Mills, Phys. Rev. 96, 191 (1954).

[3] S. Coleman and J. Mandula, Phys. Rev. 159, 1251 (1967).

[4] Th. Kaluza, Sitz. Preuss. Akad. Wiss. K1, 966 (1921);

O. Klein, Z. Physik, 37, 895 (1926).

[5] D. Freedman, S. Ferrara, and P. Van Nieuwenhuizen, Phys. Rev. D13, 3214 (1976);

S. Deser and B. Zumino, Phys. Lett. 62B, 335 (1976).

[6] M. Green and J.H. Schwartz, Phys. Lett. 149B, 117 (1984);

P. Candelas, G.T. Horowitz, A. Strominger, and E. Witten, Nucl. Phys. B258, 46 (1985);

D. Gross, J. Harvey, E. Martinec, and R. Rohm, Phys. Rev. Lett. 54, 502 (1985);

For a review and more references see: M.B. Green, J.H. Schwartz, and E. Witten, Superstring theory, Cambridge University Press 1987.

[7] E. Witten, Nucl. Phys. B186, 412 (1981).

[8] S.L. Glashow, Nucl. Phys. 22, 579 (1961); S. Weinberg, Phys. Rev. Lett. 19, 1264 (1967); A. Salam, in Proceedings of the Eight Nobel Symposium, on Elementary Particle Theory, Relativistic Groups, and Analyticit, Stochholm, Sweden, 1968, edited by N. Svartholm (Almqvist and Wikell, Stockholm, 1968).

[9] H. Georgi, in Particles and Fields- 1974, ed. C. Carlson (Amer. Inst. of Physics, New York, 1975);

H. Fritzsch and P. Minkowski, Ann. Phys. 93, 193 (1975).

[10] K. Stelle, Phys. Rev. D16, 953 (1977).

[11] B.L. Voronov and I.V. Tyutin, Yad. Fiz. (J. Nucl. Phys.) 39, 998 (1984). 